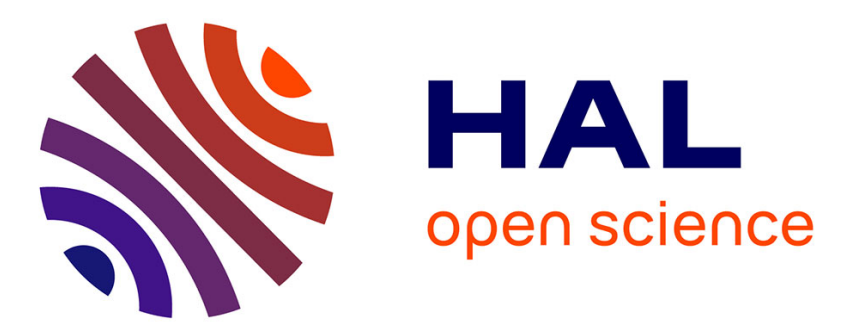

\title{
Plasma fluid modeling of microwave streamers: Approximations and accuracy
}

\author{
Emanuele Arcese, François Rogier, Jean-Pierre Boeuf
}

\section{To cite this version:}

Emanuele Arcese, François Rogier, Jean-Pierre Boeuf. Plasma fluid modeling of microwave streamers: Approximations and accuracy. Physics of Plasmas, 2017, 24 (11), pp.113517. 10.1063/1.5006651. hal-01711007

\section{HAL Id: hal-01711007 \\ https://hal.science/hal-01711007}

Submitted on 16 Feb 2018

HAL is a multi-disciplinary open access archive for the deposit and dissemination of scientific research documents, whether they are published or not. The documents may come from teaching and research institutions in France or abroad, or from public or private research centers.
L'archive ouverte pluridisciplinaire HAL, est destinée au dépôt et à la diffusion de documents scientifiques de niveau recherche, publiés ou non, émanant des établissements d'enseignement et de recherche français ou étrangers, des laboratoires publics ou privés. 


\title{
Plasma fluid modeling of microwave streamers: approximations and accuracy
}

\author{
Emanuele Arcese, ${ }^{1,2,3, \text { a) }}$ Francois Rogier, ${ }^{1}$ and Jean-Pierre Boeuf ${ }^{2}$ \\ 1) ONERA - The French Aerospace Lab, 2 Avenue douard Belin, 31000 Toulouse France \\ ${ }^{2)}$ LAPLACE (Laboratoire Plasma et Conversion d'Energie), Université de Toulouse, UPS, INPT, \\ 118 Route de Narbonne, F-31062 Toulouse Cedex 9, France \\ 3) ISAE-SUPAERO, 10 Avenue Édouard Belin, 31400 Toulouse, France
}

\begin{abstract}
Fluid models of microwave streamers at $110 \mathrm{GHz}$ in atmospheric pressure air predict the formation of filamentary plasma patterns that show good qualitative agreement with experiments. In order to perform more quantitative comparisons with experiments, we study in this paper the consequences of different types of approximations that are generally used in the fluid models. We consider here the streamer dynamics before gas heating effects become important, i.e. the first few tens of ns after breakdown at atmospheric pressure. The influence on the results of the local effective field approximation, vs local mean energy approximation is analyzed in detail. Other approximations related to the choice and method of calculation of electron transport parameters are also discussed. It is shown that the local effective field approximation is rather good for a large range of conditions of high frequency breakdown at atmospheric pressure in air while the results may be very sensitive to the choice of transport coefficients.
\end{abstract}

\section{INTRODUCTION}

Microwave breakdown in air at atmospheric pressure is characterized by the formation of localized "plasmoids" around initial seed electrons. These plasmoids elongate in the direction parallel to the applied microwave electric field because of the field and ionization enhancement at their poles, and transform into "microwave streamers" or filaments. At sufficiently high microwave frequencies (wavelength in the $\mathrm{mm}$ range) the standing wave formed in front of a microwave streamer, associated with a diffusion-ionization mechanism, leads to the generation of new streamers in front of existing ones. The successive generation of streamer filaments forms a filamentary pattern that propagates in the direction of the microwave source. These self-organized structures of filaments have been observed experimentally ${ }^{8,9,14-16}$ using a $110 \mathrm{GHz}$ pulsed gyrotron in the MW power range and predicted by physical models ${ }^{2,5,18,23,30,31}$ based on the coupling of Maxwell's equations with a simple description of the quasineutral, ambipolar plasma. The qualitative agreement between experimental measurements and simulations is rather good, but detailed quantitative comparisons are still to be done.

In this paper we focus on the different approximations affecting the accuracy of the simulations, in order to prepare more quantitative comparisons between experiments and models. An important approximation of previous physical models is the local effective field approximation where the ionization frequency characterizing the growth of the plasmoid and of the streamer is supposed to depend on a local effective field defined in the next section. Because of the large density gradients at the tips of a microwave streamer, the local effective field approximation is questionable and we have developed a more accurate model where the local effective field approxima-

a) Electronic mail: emanuele.arcese@onera.fr tion is replaced by a local mean energy approximation, i.e. where an energy equation is taken into account (assuming that the electron distribution function for a given mean electron energy is the same as that obtained under a uniform electric field and that the reaction rates are functions of the local mean electron energy). In this paper we consider conditions where the wave frequency is larger than the electron energy exchange frequency and much larger than the ionization frequency. Therefore the modulation of the electron mean energy and ionization frequency within one cycle is small and the electron energy absorption and loss terms in the energy equations are integrated in time. The "non-locality" discussed in this paper is a non-locality in space. In contrast with the local effective field approximation, the local mean energy approximation is able to take into account a finite (nonzero) energy relaxation length and/or time, and has been used in many discharges models ${ }^{3,10,11,22,23,29}$. Other approximations inherent to the fluid models are related to the transport coefficients and collision rates that are used and to their dependence on the local effective field or mean electron energy. In this paper we define a parameter that characterizes the "non-locality" of the energy equation, and we perform detailed comparisons of microwave breakdown and filamentary pattern formation as predicted by models based on the local effective field or local mean energy approximation. We show that local effective field and local mean energy models give very similar results under conditions of microwave breakdown at high pressure and high frequency. We show, on the other hand that the results are sensitive to other approximations related to the transport coefficients used in the model. In the following, the two models are either called local effective field and local mean energy models or local and non-local models (since the local mean energy model takes into account the non-locality of the electron energy balance).

The paper is organized as follows. The Maxwellplasma model and the local effective field and local mean energy approximations are described in section II. In sec- 
tion III we discuss the choice of transport coefficients and approximations used in the models. In section IV we compare the local effective field and local mean energy approximation and define a parameter that characterizes the "non-locality" of the problem. The conditions of the simulations and some aspects of the numerical method are presented in section $\mathrm{V}$. The results of the simulations and the consequences of the different approximations are analyzed in section VI

\section{PHYSICAL MODEL}

The physical model describing the dynamics of microwave streamers consists of plasma transport equations coupled with Maxwell's equations via the electron current density. Particularly, microwave oscillations are described by Maxwell's equations:

$$
\begin{aligned}
\nabla \times \tilde{\mathbf{E}} & =-\frac{\partial \tilde{\mathbf{B}}}{\partial t} \\
\nabla \times \tilde{\mathbf{B}} & =\mu_{0}\left(\tilde{\mathbf{J}}+\epsilon_{0} \frac{\partial \tilde{\mathbf{E}}}{\partial t}\right)
\end{aligned}
$$

where $\epsilon_{0}$ and $\mu_{0}$ are the free space permittivity and permeability respectively. $\tilde{\mathbf{J}}$ is the electron current density through which the coupling with plasma dynamics is established:

$$
\tilde{\mathbf{J}}=-e n_{e} \tilde{\mathbf{u}}_{e}
$$

Due to the relatively large ion mass the ion motion is neglected with respect to the electron motion. The instantaneous electron velocity $\tilde{\mathbf{u}}_{e}$ is determined by the approximate electron momentum transfer equation:

$$
\frac{\partial \tilde{\mathbf{u}}_{e}}{\partial t}=-\frac{e \tilde{\mathbf{E}}}{m_{e}}-\nu_{m} \tilde{\mathbf{u}}_{e}
$$

where $m_{e}$ is the electron mass and $\nu_{m}$ the electronneutral momentum transfer frequency. It is important to note that the instantaneous electron velocity changes over the microwave time scale according to electric field variations (all quantities varying over the wave cycle are denoted with tilde) while the plasma properties, such as the electron density $n_{e}$, the time averaged electron mean energy, etc, are assumed to be constant during the microwave field period. Indeed we average the transport of plasma quantities over one wave cycle as the time scale of plasma evolution is much longer than that of the microwave period. Note that the mean electron velocity used in Maxwell's equation is obtained from a momentum transfer equation neglecting density gradients and has a zero time average while the mean electron velocity used in the plasma model below is time averaged but take into account the density gradient and associated diffusion (which is responsible for charged particles transport in this context).

The electron density in $\tilde{\mathbf{J}}$ is solution of the averaged plasma transport equations. In this context, two plasma fluid models of different order are considered. On the one hand the model based on the local effective field approximation and on the other hand the model based on the local mean energy approximation. These approximations determine the accuracy of the related fluid model as they are derived from kinetic assumptions essentially made on the form of the electron distribution function. In the following subsections a detailed analysis of the two fluid models is carried out.

\section{A. First-order model}

The first-order model proposed by Boeuf et $a l .{ }^{2}$ describes the conservation of the number of electrons in a quasi-neutral microwave plasma:

$$
\begin{gathered}
\frac{\partial n_{e}}{\partial t}+\nabla \cdot \boldsymbol{\Gamma}=\nu_{e f f} n_{e}-r n_{e}^{2} \\
\boldsymbol{\Gamma}=n_{e} \mathbf{u}_{e}=-D_{e f f} \nabla n_{e} .
\end{gathered}
$$

$\mathbf{u}_{e}$ is the time averaged electron mean velocity. The source term on the right hand side of the continuity equation represents the net number of electrons created per unit time per unit volume. In our case, $\nu_{e f f}$ is an effective ionization coefficient taking into account the electron-impact ionization and attachment processes $\left(\nu_{e f f}=\nu_{i}-\nu_{a}\right)$ and $r$ is the electron-ion recombination coefficient. Using the well known local effective field approximation and the effective field concept ${ }^{21}$ the effective ionization frequency is only expressed as a function of the local reduced effective electric field $E_{e f f} / N$ where

$$
E_{e f f}=E_{r m s}\left(1+\omega^{2} / \nu_{m}^{2}\right)^{-1 / 2}
$$

with $\omega=2 \pi f$ the wave angular frequency and $E_{r m s}$ the root-mean-square (rms) value of the varying electric field. Such an approximation reflects the scenario of collisional particles where energy gain, from the electric field, collisional momentum and energy losses are locally balanced.

Concerning the left-hand side of equation 4 the term inside the divergence operator $\boldsymbol{\Gamma}$ represents the flux of electrons. Its diffusion form is obtained by approximating the general momentum transfer equation:

$\frac{\partial\left(n_{e} \mathbf{u}_{e}\right)}{\partial t}+\nabla \cdot\left(n_{e} \mathbf{u}_{e} \otimes \mathbf{u}_{e}\right)+\frac{1}{m_{e}} \nabla \cdot \underline{\mathbf{P}_{\mathbf{e}}}+\frac{e n_{e}}{m_{e}} \mathbf{E}=-n_{e} \nu_{m} \mathbf{u}_{e}$

First, a standard closure approximation is employed on the pressure tensor. It is considered diagonal and 
isotropic such that $\nabla \cdot \underline{\mathbf{P}_{\mathbf{e}}}=\nabla\left(n_{e} k_{B} T_{e}\right)=\nabla p\left(k_{B}\right.$ and $T_{e}$ are the Boltzmann constant and the electron temperature respectively), i.e. the force per unit volume due to the variation of electron velocities is given by the gradient of the scalar pressure. Second, the inertia terms of equation 6 are neglected with respect to the collision term since collisions are assumed to take place on shorter time and length scales than macroscopic variations. As a result a simplified momentum transfer equation is obtained. The electron flux is thus only given by the drift and diffusion terms:

$n_{e} \mathbf{u}_{e}=-\frac{e n_{e} \mathbf{E}}{m_{e} \nu_{m}}-\frac{k_{B}}{m_{e} \nu_{m}} \nabla\left(n_{e} T_{e}\right) \approx-n_{e} \mu_{e} \mathbf{E}-D_{e} \nabla n_{e}$.

The two transport coefficients $\mu_{e}=e /\left(m_{e} \nu_{m}\right)$ and $D_{e}=k_{B} T_{e} /\left(m_{e} \nu_{m}\right)$ are the electron mobility and the electron free diffusion coefficient respectively which are linked via the Einstein relation $D_{e} / \mu_{e}=k_{B} T_{e} / e$ under the assumption of Maxwellian electrons. The diffusion term (the second term in equation 7) is approximated such that only the gradient of electron density is kept as the electron temperature variations are truly small compared to those of the electron density.

Since the transport of plasma quantities is averaged over one wave cycle the electric field appearing in equation 7 only represents the space charge field: $\mathbf{E}=\mathbf{E}_{s p}$ (we neglect the contribution of the magnetic force). Such an electric field is normally obtained by solving the Poisson's equation coupled with ion and electron continuity equations. In our case it is reasonable to assume quasineutrality because, 1), the plasma size is much larger than the Debye length, and, 2), there is not net transport due to charged particle drift in the oscillating sheath field. Therefore, by imposing locally the equality of electron and ion densities $\left(n_{e}=n_{i}\right)$ the space charge effects are described by an ambipolar field maintaining equal electron and ion fluxes:

$$
\boldsymbol{\Gamma}=-\frac{\mu_{i} D_{e}+\mu_{e} D_{i}}{\mu_{i}+\mu_{e}} \nabla n_{e}=-D_{a} \nabla n_{e} .
$$

$D_{a}$ is the ambipolar diffusion coefficient and $\mu_{i}$ and $D_{i}$ are the ion mobility and ion free diffusion coefficient respectively. The ambipolar diffusion occurs in the bulk of plasma while at its edge electrons diffuse freely. In order to properly describe the transition from ambipolar to free diffusion a heuristic effective diffusion coefficient $D_{\text {eff }}$ is introduced:

$$
D_{\text {eff }}=\frac{\alpha D_{e}+D_{a}}{1+\alpha}
$$

with $\alpha=\nu_{i} \tau_{M}$ and $\tau_{M}$ is the Maxwell relaxation time given by $\tau_{M}=\epsilon_{0} /\left(e n_{e}\left(\mu_{i}+\mu_{e}\right)\right)$. The derivation of this coefficient is given by Boeuf et al. ${ }^{2,31}$. Therefore, using $D_{\text {eff }}$ in the common flux density we obtain the final form of $\boldsymbol{\Gamma}$ (given by the second equation of 4 ). In this context, we will refer to this first-order model as the local model.

\section{B. Second-order model}

The second-order model we propose is derived by adding the third velocity moment of the electron Boltzmann equation and reads in its final form as:

$$
\begin{aligned}
& \frac{\partial n_{e}}{\partial t}-\nabla \cdot\left(D_{e f f} \nabla n_{e}\right)=\nu_{e f f} n_{e}-r n_{e}^{2} \\
& \frac{\partial n_{\varepsilon}}{\partial t}-\frac{5}{3} \nabla \cdot\left(D_{e f f} \nabla n_{\varepsilon}\right)=-n_{e}\left(e<\tilde{\mathbf{u}}_{e} \cdot \tilde{\mathbf{E}}>_{T_{M}}+\Theta\right)
\end{aligned}
$$

where $n_{\varepsilon}=n_{e} \varepsilon_{e}$ is the energy density, $\varepsilon_{e}$ is the mean electron energy and $\Theta$ is the mean power loss per electron. It is essentially a system of conservation laws for electron density and energy averaged over a microwave field period.

$\Theta$ expresses the energy losses during inelastic and elastic collisions. We consider in this paper only collisions with molecules in the ground state so $\Theta$ can be explicitly written as:

$$
\Theta=\sum_{\alpha} n_{\alpha}\left(\sum_{j} \xi_{j, \alpha} k_{j, \alpha}+\frac{2 m}{M_{\alpha}} \varepsilon_{e} k_{m, \alpha}\right) .
$$

with $n_{\alpha}$ and $M_{\alpha}$ the density and mass of the $\alpha$ gas component, $k_{j, \alpha}$ the rate coefficients for inelastic collisions including ionization, $\xi_{j, \alpha}$ the threshold energy of each inelastic collision and $k_{m, \alpha}$ the momentum rate coefficients. Introducing the concept of energy relaxation frequency $\nu_{\varepsilon}$ (a frequency taking into account the rate coefficients related to elastic and inelastic collisions) we can express the energy losses (11) in a more concise way: $\Theta=\nu_{\varepsilon} \varepsilon_{e}$.

The macroscopic description of these coefficients strongly depends on the assumptions made on the electron distribution function. This time, we employ the local mean energy approximation which expresses the rate coefficients, hence the effective ionization and transport coefficients, as a function of the local mean electron energy $\varepsilon_{e}$ (instead of a function of the local effective field as in the local effective field approximation).

The source term of the energy density equation (10) also contains the term $-e<\tilde{\mathbf{u}}_{e} \cdot \tilde{\mathbf{E}}>_{T_{M}}$, the mean energy gained per unit time by an electron. The instantaneous quantity $\left(\tilde{\mathbf{u}}_{e} \cdot \tilde{\mathbf{E}}\right)$ is averaged over the entire microwave period $T_{M}=2 \pi / \omega$ in order to coherently obtain a mean power:

$$
-e<\tilde{\mathbf{u}}_{e} \cdot \tilde{\mathbf{E}}>_{T_{M}}=-\frac{e}{T_{M}} \int_{0}^{T_{M}} \tilde{\mathbf{u}}_{e} \cdot \tilde{\mathbf{E}} d t .
$$

Its value essentially depends on the phase shift between the instantaneous electron velocity and the microwave field. Note that this mean power could be described, as in the previous local effective field approximation model, by 
the effective field (equation 5). In this paper, we actually use a slightly more accurate estimation of the absorbed power (see section IV).

The energy equation in (10) is obtained by a usual simplification of the general energy equation:

$$
\frac{\partial\left(n_{e} \varepsilon_{e}\right)}{\partial t}+\nabla \cdot\left(n_{e} \mathbf{u}_{e} \varepsilon_{e}+\underline{\mathbf{P}} \cdot \mathbf{u}_{e}+\mathbf{q}\right)=-n_{e}\left(e \mathbf{u}_{e} \cdot \mathbf{E}+\Theta\right) .
$$

The same assumptions as in the momentum transfer equation are made for the pressure tensor and electron flux. The kinetic energy is neglected with respect to the thermal energy so that $\varepsilon_{e}=3 k_{B} T_{e} / 2$ (since the distribution is not Maxwellian this equation defines the electron temperature $T_{e}$ ). The heat flux vector $\mathbf{q}$ is assumed to $\mathrm{be}^{1}$ :

$$
\mathbf{q}=-\frac{5}{2} k_{B} D_{e f f} n_{e} \nabla T_{e} .
$$

Such an approximation is essentially derived from the perturbation solution of the electron Boltzmann equation around a local Maxwellian electron distribution function assuming a constant kinetic pressure $\left(n_{e} k_{B} T_{e}=\right.$ const $)$. Finally, the first term in the right-hand side of equation 13 is treated as follows. In a microwave plasma, this term is normally made of two quantities: the first one related to an important energy gain due to the mean work of the microwave field $\left(-e n_{e}<\tilde{\mathbf{u}}_{e} \cdot \tilde{\mathbf{E}}>_{T_{M}}\right)$ and the second one related to energy losses (diffusion cooling) due to the work of the ambipolar field $\left(-e n_{e} \mathbf{u}_{e} \cdot \mathbf{E}_{s p}\right)$. As we assumed quasi-neutrality, $\mathbf{E}_{s p}$ is the ambipolar field and can be written as ${ }^{2}$

$$
\mathbf{E}_{s p} \approx-\frac{1}{1+\alpha} \frac{D_{e}}{\mu_{e}} \frac{\nabla n_{e}}{n_{e}} .
$$

Practically, the electron diffusion cooling proves negligible compared to the electron power loss due to collisions both in the bulk and in the plasma front:

$$
e \mathbf{u}_{e} \cdot \mathbf{E}_{s p} \approx \frac{2}{3} \frac{D_{e f f}}{1+\alpha} \varepsilon_{e}\left(\frac{\nabla n_{e}}{n_{e}}\right)^{2} \ll \Theta
$$

as the upper bound of the diffusion cooling term is $\nu_{i} \varepsilon_{e}$ (note that $L=\left|\nabla n_{e} / n_{e}\right|^{-1}=\left(D_{e} / \nu_{i}\right)^{1 / 2}$ is the characteristic length of the propagating front under constant diffusion coefficient and ionization frequency ${ }^{5}$ ) and in our conditions $\nu_{i}$ is much smaller than $\nu_{\varepsilon}$ (see Figures 1 and 2 ). Under these assumptions we obtain the final form of the second-order model (10) which will be called the non-local model in what follows.

We note that the local mean energy approximation is much more realistic than the local effective field approximation since it allows energy transport from regions where electrons are strongly heated by the fields to regions where they are not. This may have important (even qualitative) consequences in many discharges and is a usual situation at lower pressures where the energy relaxation length may not be small with respect to the spatial scale of plasma and field non-uniformities.

\section{TRANSPORT AND RATE COEFFICIENTS}

An essential aspect of the models is related to the choice of transport coefficients and rate coefficients. These coefficients and their dependence on the reduced electric field or mean electron energy can be obtained from solutions of the Boltzmann equation under a uniform field. Then the values of these coefficients rely on the choice of the electron-neutral cross-sections. In the present model of microwave discharges in air (assuming a $0.78 \mathrm{~N}_{2}-0.21 \mathrm{O}_{2}-0.01 \mathrm{Ar}$ mixture composition) we chose to use the cross-section data set of Biagi in the LXCat database $^{27}$ which provides, according to Kourtzanidis et al. ${ }^{19}$ the most accurate breakdown field prediction in air.

Another question is the derivation of the transport and rate coefficients and their variations with the applied field at a given pressure and microwave frequency. In this paper we do not use the effective field concept defined in section II. A more consistent and more accurate method is to use the solver BOLSIG $+{ }^{25}$, which offers an option, in the case of high frequency fields, to solve the Boltzmann equation by taking into account the oscillations of the field over one cycle. This option provides the cycle averaged electron distribution function, transport and rate coefficients as a function of the reduced electric field $E / N$ and the reduced angular frequency $\omega / N$. In the case of the local mean energy approximation, the transport and rate coefficients can be tabulated as a function of mean electron energy $\varepsilon_{e}$ and reduced angular frequency $\omega / N$.

As we are dealing with oscillating fields, the electron mobility is identified by a complex quantity $\mu_{e}=$ $\mu_{r e}+j \mu_{i m}$ according to the definition of the electron drift velocity in high frequency fields ${ }^{12}$. The real part only determines the net power absorption and the imaginary part is necessary to determine the proper value of the momentum transfer collision frequency $\nu_{m}$ related to given values of $E / N$ and $\omega / N$ (or $\varepsilon_{e}$ and $\omega / N$ in the local mean energy approximation). Indeed in the BOLSIG + solver, $\nu_{m}$ is not directly obtained by the cross sections and its value can be deduced from the knowledge of the averaged electron mobility $\mu_{e}$ through the following equations:

$$
\begin{aligned}
\mu_{r e}(E / N, \omega / N) & =\frac{e \nu_{m}}{m_{e}\left(\nu_{m}^{2}+\omega^{2}\right)} \\
\mu_{i m}(E / N, \omega / N) & =-\frac{e \omega}{m_{e}\left(\nu_{m}^{2}+\omega^{2}\right)} .
\end{aligned}
$$

The ion mobility is taken as a constant equal to $\mu_{\text {re }}\left(E_{\text {inc }} / N, \omega / N\right) / 200$, i.e. about 200 times smaller than the electron mobility corresponding to the reduced incident electric field and reduced angular frequency. 
The free electron and ambipolar diffusion coefficients are given by $D_{e}=k_{B} T_{e} \mu_{r e} / e$ and $D_{a}=k_{B} T_{e} \mu_{i} / e$ (the ion free diffusion coefficient is simply set to zero $D_{i}=0$ ). Figures 1 and 2 show a typical trend of the reduced effective ionization frequency, the reduced energy relaxation frequency and the reduced momentum transfer collision frequency used in the models for air as a function of the reduced electric field at different reduced wave angular frequencies.

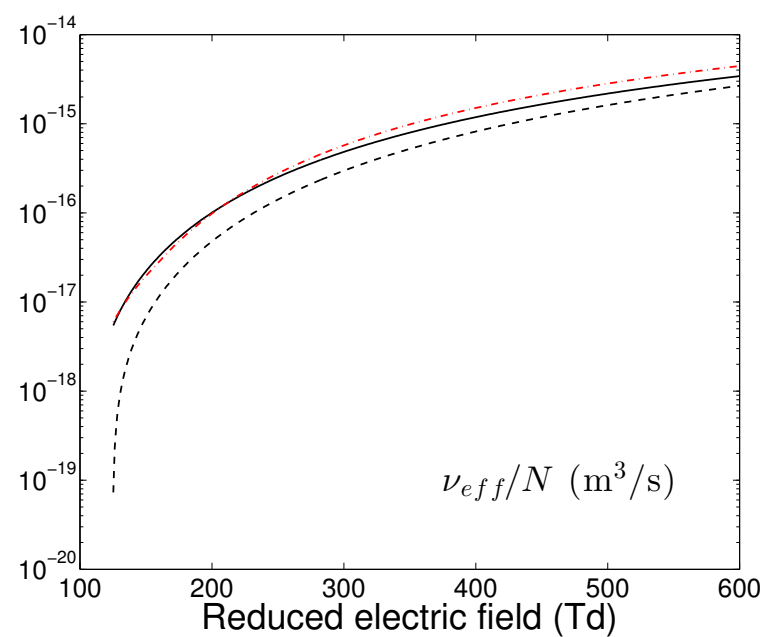

FIG. 1. Reduced effective ionization frequency $\nu_{\text {eff }} / N$ in logarithmic scale for air as function of the reduced electric field $E_{r m s} / N$. They are obtained at $\omega=2 \pi 110 \times 10^{9} \mathrm{rad} / \mathrm{s}$, $p=760$ torr (solid line) and $\omega=2 \pi 110 \times 10^{9} \mathrm{rad} / \mathrm{s}, p=200$ torr (dashed line) from the BOLSIG+ solver using the Biagi input data. The reduced effective ionization frequency used by Chaudhury et al. ${ }^{5}$ for air at $\omega=2 \pi 110 \times 10^{9} \mathrm{rad} / \mathrm{s}, p=760$ torr is added as comparison (red dash-dot line).

Some further remarks have to be made on the momentum transfer collision frequency and the diffusion coefficient. In many theoretical and numerical studies $\nu_{m}$ is estimated as function of the only ambient pressure using the simple equation ${ }^{21}: \nu_{m} / p=5.3 \times 10^{9} \mathrm{~s}^{-1}$ torr $^{-1}$ (e.g. $\nu_{m} / N=1.65 \times 10^{-13} \mathrm{~m}^{3} / \mathrm{s}$ at atmospheric pressure, refer to Fig. 2) because of its weak dependence with respect to the electric field. In this paper the momentum transfer collision frequency is obtained as described above, as function of $E / N$ and $\omega / N$. The consequence of taking $\nu_{m}$ constant or not with the electric field in microwave discharge modeling is estimated in the results section VI.

The electron diffusion coefficient is a function of $E / N$ and $\omega / N$ in the local effective field approximation, or of $\varepsilon_{e}$ and $\omega / N$ in the local mean energy approximation. In several published models of microwave plasma at atmospheric pressure $2,4-6,18,20,26,30,31$ the electron temperature in the diffusion coefficient is taken as a constant equal to $2 \mathrm{eV}$. In the paper of Woo et al. ${ }^{28}$ the electron temperature is expressed as a function of the effective field as:
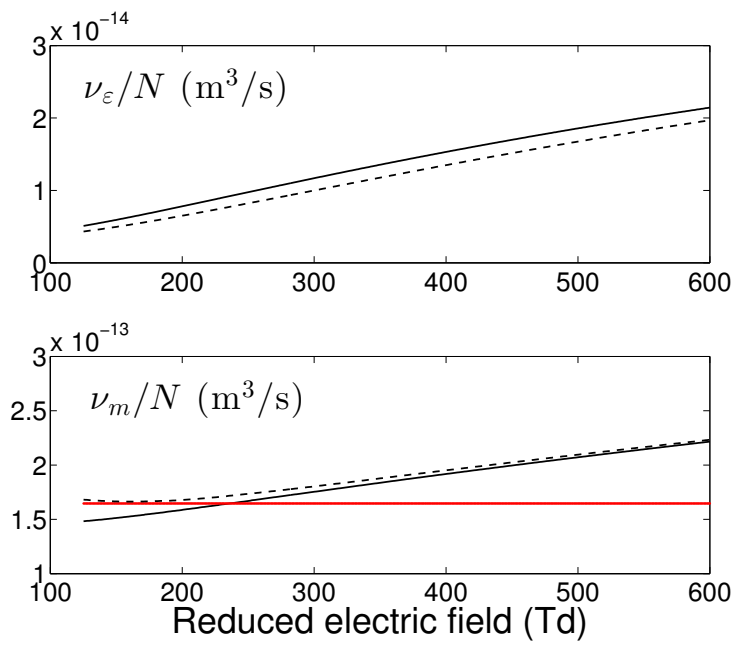

FIG. 2. (Color online) Reduced energy relaxation frequency $\nu_{\varepsilon} / N$ (top figure) and reduced momentum transfer collision frequency $\nu_{m} / N$ (bottom figure) for air as function of the reduced electric field $E_{r m s} / N$. They are obtained at $\omega=$ $2 \pi 110 \times 10^{9} \mathrm{rad} / \mathrm{s}, p=760$ torr (solid line) and $\omega=2 \pi 110 \times$ $10^{9} \mathrm{rad} / \mathrm{s}, p=200$ torr (dashed line) from the BOLSIG+ solver using the Biagi input data. In the bottom figure, the constant reduced momentum transfer collision frequency used by Chaudhury et al. ${ }^{5}$ for air at $p=760$ torr is added as comparison, $\nu_{m} / N=1.65 \times 10^{-13} \mathrm{~m}^{3} / \mathrm{s}$ (red solid line).

$$
\frac{k_{B} T_{e}}{e}=\left[2.1 \times 10^{-3} \frac{E_{\text {eff }}}{p}\left(91.0+\frac{E_{e f f}}{p}\right)\right]^{1 / 3}
$$

where $p$ is the gas pressure in torr and $E_{\text {eff }}$ is in $\mathrm{V} / \mathrm{cm}$. In the present paper we use a functional dependence of the mean electron energy, $\varepsilon_{e}=\varepsilon_{e}(E / N, \omega / N)$, obtained from BOLSIG+ using the Biagi data set of cross-sections. This gives significantly different variations of the electron temperature with the effective field, as can be seen on Fig. 3 which compares the electron temperature obtained with the Biagi data set with that from 18.

We will see in the the simulation results presented in section VI, that the use of a constant mean electron energy leads to an erroneous estimation of the propagation velocity of the plasma front.

Finally, regarding the electron-ion recombination we simply assume (see for instance Ref. ${ }^{17}$ ): $r=\alpha \times$ $10^{-13}\left(300 / T_{e}\right)^{-1 / 2} \mathrm{~m}^{-3} \mathrm{~s}^{-1}$, where $T_{e}$ is in Kelvin and $0 \leq \alpha \leq 2$ is set to 0.1 according to Ref. ${ }^{5}$.

\section{LOCAL EFFECTIVE FIELD VS. LOCAL MEAN ENERGY APPROXIMATIONS}

In this section we compare the local effective field and local mean energy models and study the conditions of validity of the local effective field model. By re-scaling 


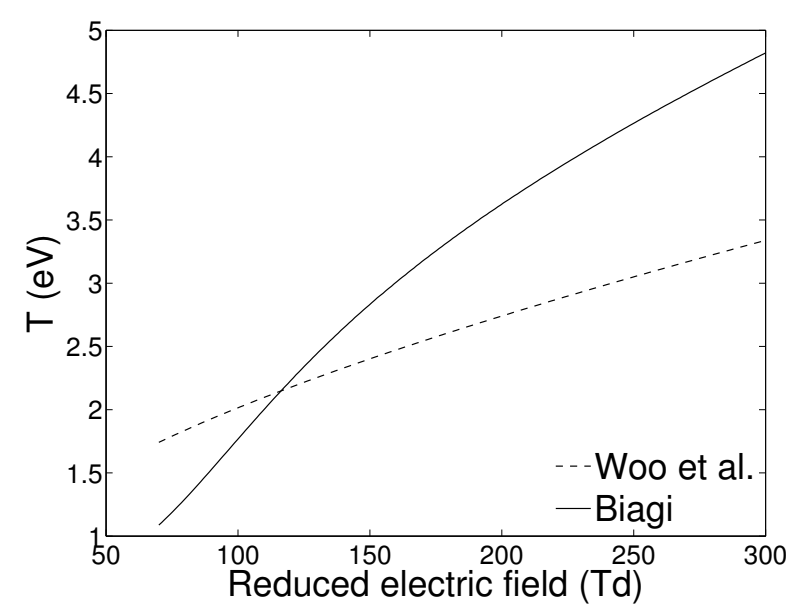

FIG. 3. Electron temperature $T_{e}$ as function of the reduced electric field $E_{r m s} / N$. Temperature trends obtained by means of equation 18 (dashed line) and BOLSIG+ solver using the Biagi input data (solid line).

the local mean energy model, we derive a parameter that characterizes the "non-locality" of the problem in the microwave breakdown context.

In order to simplify calculations and make the analysis more clear, let us assume to be at the front of plasma $\left(D_{e f f}=D_{e}\right)$ and neglect the recombination coefficient and the attachment frequency $\left(r=0\right.$ and $\left.\nu_{e f f}=\nu_{i}\right)$. The referred-to assumptions do not essentially influence the principal result and the analysis can however be generalized by taking into account the complete non-local model. Therefore, the related model is:

$$
\begin{aligned}
& \frac{\partial n_{e}}{\partial t}-\nabla \cdot\left(D_{e} \nabla n_{e}\right)=\nu_{i} n_{e} \\
& \frac{\partial \varepsilon_{e}}{\partial t}-\frac{5}{3} \frac{\nabla}{n_{e}} \cdot\left(D_{e} \nabla n_{\varepsilon}\right)+\varepsilon_{e} \frac{\nabla}{n_{e}} \cdot\left(D_{e} \nabla n_{e}\right)= \\
& =-\left(e<\tilde{\mathbf{u}}_{e} \cdot \tilde{\mathbf{E}}>_{T_{M}}+\varepsilon_{e}\left(\nu_{\varepsilon}+\nu_{i}\right)\right)
\end{aligned}
$$

where the explicit form of the mean electron energy equation is here considered (note that $\partial_{t} n_{\varepsilon}=n_{e} \partial_{t} \varepsilon_{e}+$ $\left.\varepsilon_{e} \partial_{t} n_{e}\right)$. Some characteristics parameters have to be defined (these parameters will have the bar on the top) in our problem in order to properly make the model equations dimensionless. For this purpose, we look at a characteristic scenario where the properties of microwaves and gas are fixed (i.e. the electric field, the field frequency and the gas density are set to given values $\bar{E}_{r m s}$, $\bar{\omega}, \bar{N}$ respectively) and the condition expressing the local effective field approximation is fulfilled (a typical profile of plasma density, $\bar{n}_{e}$, is assumed given). Particularly, in such a condition the mean power absorbed by electrons from the wave field is exactly balanced by the power loss due to elastic and inelastic collisions. By looking for the steady state of the mean electron energy $\left(\partial_{t} \varepsilon_{e}=0\right)$ in a uniform medium it is straightforward to express the above condition as (from system 19):

$$
-e<\tilde{\mathbf{u}}_{e} \cdot \tilde{\mathbf{E}}>_{T_{M}}=\varepsilon_{e}\left(\nu_{\varepsilon}+\nu_{i}\right)
$$

We therefore recognize that the mean electron energy is only function of the reduced electric field $E_{r m s} / N$ and the reduced field frequency $\omega / N$ (as the collision and ionization frequencies are proportional to the gas density). In this case a characteristic energy can be identified:

$$
\bar{\varepsilon}_{e}=\frac{e^{2}}{m_{e} \bar{\nu}_{m}\left(\bar{\nu}_{\varepsilon}+\bar{\nu}_{i}\right)} \frac{\bar{E}_{r m s}^{2}}{\left(1+\frac{\bar{\omega}^{2}}{\bar{\nu}_{m}^{2}}\right)} .
$$

Note that once the reduced electric field and field frequency are given all transport and rate coefficients are consequently determined even the electron diffusion coefficient through the mean electron energy knowledge. Moreover, the characteristic mean power absorbed by electrons (hence the characteristic loss mean power equivalently, $\left.\bar{\varepsilon}_{e}\left(\bar{\nu}_{\varepsilon}+\bar{\nu}_{i}\right)\right)$ is:

$$
-e<\tilde{\mathbf{u}}_{e} \cdot \tilde{\mathbf{E}}>_{T_{M}}=\frac{e^{2}}{m_{e} \bar{\nu}_{m}} \frac{\bar{E}_{r m s}^{2}}{\left(1+\frac{\bar{\omega}^{2}}{\bar{\nu}_{m}^{2}}\right)} .
$$

Let us then set the characteristic lengths and time of the two-dimensional phenomenon as:

$$
\bar{x}=\bar{y}=L=\sqrt{\frac{\bar{D}_{e}}{\bar{\nu}_{i}}} ; \quad \bar{t}=\frac{L}{v_{f}}=\frac{1}{2 \bar{\nu}_{i}} .
$$

where $L$ and $v_{f}=2\left(\bar{D}_{e} \bar{\nu}_{i}\right)^{1 / 2}$ are the characteristic length and velocity respectively of the propagating front that is solution of the first equation of 19 under constant diffusion coefficient and ionization frequency ${ }^{5}$. Once all characteristic parameters are identified, a change of variables in equations 19 is introduced:

$$
x \rightarrow L x ; y \rightarrow L y ; t \rightarrow \bar{t} t ; \varepsilon_{e} \rightarrow \bar{\varepsilon}_{e} \varepsilon_{e} ; D_{e} \rightarrow \bar{D}_{e} D_{e} ; \ldots
$$

The re-scaled transport equations of the electron density and the electron mean energy are:

$$
\begin{aligned}
\frac{\bar{n}_{e}}{\bar{t}} \frac{\partial n_{e}}{\partial t}= & \frac{\bar{D}_{e} \bar{n}_{e}}{L^{2}} \nabla \cdot\left(D_{e} \nabla n_{e}\right)+\bar{\nu}_{i} \bar{n}_{e} \nu_{i} n_{e} \\
\frac{\bar{\varepsilon}_{e}}{\bar{t}} \frac{\partial \varepsilon_{e}}{\partial t}= & \frac{5}{3} \frac{\bar{D}_{e} \bar{\varepsilon}_{e}}{L^{2}} \frac{\nabla}{n_{e}} \cdot\left(D_{e} \nabla n_{\varepsilon}\right)-\frac{\bar{D}_{e} \bar{\varepsilon}_{e}}{L^{2}} \varepsilon_{e} \frac{\nabla}{n_{e}} \cdot\left(D_{e} \nabla n_{e}\right)+ \\
& -\frac{e^{2}}{m_{e} \bar{\nu}_{m}} \frac{\bar{E}_{r m s}^{2}}{\left(1+\frac{\bar{\omega}^{2}}{\bar{\nu}_{m}^{2}}\right)}\left(e<\tilde{\mathbf{u}}_{e} \cdot \tilde{\mathbf{E}}>_{T_{M}}+\right. \\
& \left.+\varepsilon_{e}\left(\nu_{\varepsilon}+\nu_{i}\right)\right) .
\end{aligned}
$$

Dividing the first and the second equation by $\bar{n}_{e} / \bar{t}$ and $\bar{\varepsilon}_{e} / \bar{t}$ respectively and grouping similar terms we finally obtain the dimensionless form of the non-local model 19: 


$$
\begin{aligned}
& \frac{\partial n_{e}}{\partial t}-\frac{\nabla}{2} \cdot\left(D_{e} \nabla n_{e}\right)=\frac{1}{2} \nu_{i} n_{e} \\
& \frac{\partial \varepsilon_{e}}{\partial t}-\frac{5}{6} \frac{\nabla}{n_{e}} \cdot\left(D_{e} \nabla n_{\varepsilon}\right)+\frac{\varepsilon_{e}}{2} \frac{\nabla}{n_{e}} \cdot\left(D_{e} \nabla n_{e}\right)= \\
& =-\frac{1}{2 \delta}\left(e<\tilde{\mathbf{u}}_{e} \cdot \tilde{\mathbf{E}}>_{T_{M}}+\varepsilon_{e}\left(\nu_{\varepsilon}+\nu_{i}\right)\right) .
\end{aligned}
$$

A dimensionless parameter $\delta$ appears in the source term of the second equation. It is defined as:

$$
\delta=\frac{m_{e}}{e^{2}} \frac{\bar{\varepsilon}_{e} \bar{\nu}_{m} \bar{\nu}_{i}\left(1+\frac{\bar{\omega}^{2}}{\bar{\nu}_{m}^{2}}\right)}{\bar{E}_{r m s}^{2}}=\frac{\bar{\nu}_{i}}{\bar{\nu}_{\varepsilon}+\bar{\nu}_{i}}
$$

and expresses the ratio between the mean energy that electrons have in a given condition and the work done by the corresponding wave field on these electrons. Roughly speaking, it represents the discrepancy between the local effective field and the local mean energy approximations. In fact, the local effective field regime appears as an asymptotic limit of the local mean energy model as $\delta \rightarrow 0$ (in this case, the second equation of 26 exactly tends to condition 20 and the non-local model, 26, reduces to the local model). On the other hand, the more $\delta$ increases the more the electron energy transport becomes important and the local effective field approximation is no longer sufficient to describe the physical phenomenon.

We can deduce the functional dependency of $\delta: \delta=$ $\delta(E / N, \omega / N)$, and that $0 \leq \delta<1$. Figure 4 shows the parameter $\delta$ over common ranges of $E / N$ and $\omega / N$ for microwave discharges in air. We find that $\delta$ strongly de-

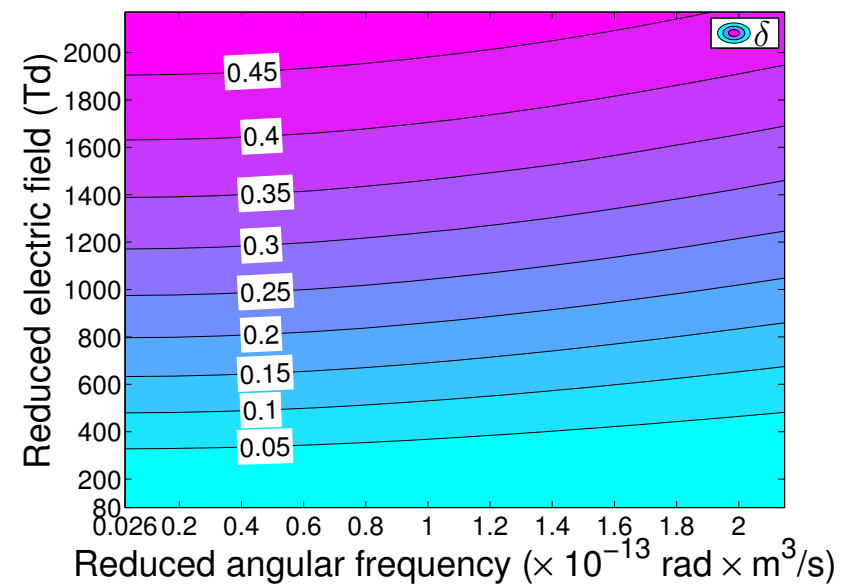

FIG. 4. (Color online) Parameter $\delta=\nu_{i} /\left(\nu_{\varepsilon}+\nu_{i}\right)$ calculated as function of $E_{r m s} / N$ and $\omega / N$ (note that at $110 \mathrm{GHz}$ and atmospheric pressure, $p=760$ torr, $\omega / N=0.2825 \times 10^{-13}$ $\operatorname{rad} \times \mathrm{m}^{3} / \mathrm{s}$ ). Calculations are performed using the BOLSIG+ solver with the Biagi input data.

pends on the reduced electric field and slightly on the reduced angular frequency. Specifically, in high collisional conditions (e.g. as $\omega / N<0.8 \times 10^{-13} \mathrm{rad} \times \mathrm{m}^{3} / \mathrm{s}$, namely, in the left side of the graph of Fig. 4) $\delta$ is almost constant with $\omega / N$. Thus, the discrepancy of the two fluid approximations essentially depends on the reduced electric field, $\delta \approx \delta(E / N)$. Otherwise, as the field angular frequency is comparable or even higher than the momentum transfer collision frequency, the reduced angular frequency variations affect much more the models discrepancy, thus $\delta=\delta(E / N, \omega / N)$.

Note that the graph in Fig. 4 and hence our parameter $\delta$ does not establish the precise conditions at which the local model is physically valid or not, but it points out how the two fluid approximations differ as function of microwave and gas conditions.

\section{SIMULATION CONDITIONS AND NUMERICAL METHODS}

The objective of the following simulations is to assess the accuracy of the above plasma fluid approximations (hence the concept expressed by the dimensionless parameter $\delta$ ) and some related physical assumptions as previously mentioned by numerically comparing the plasma models on a same case of microwave discharge. For this purpose we focus on the formation and propagation of the plasma pattern during high-frequency microwave breakdown in air in overcritical field conditions. A complex dynamics already well observed in recent experiments ${ }^{9,15,24}$.

The simulations are two-dimensional and we consider plane waves where the electric field is in the simulation domain and the magnetic field is perpendicular to the domain. The computational domain is dived into a scattered field (SF) and a total field (TF) domain (which size is $2.5 \lambda \times 2 \lambda$, where $\lambda$ is the wavelength) in order to inject the transverse electric (TE) waves (from left to right). Boundary conditions for electromagnetic waves surround then the global domain. In our calculations the microwave beam is not focused but we use an initial electron density profile of Gaussian type with a maximum of $10^{15} \mathrm{~m}^{-3}$ and a standard deviation of $50 \mu \mathrm{m}$ centered at predefined breakdown spot. The spot position is on the central X-axis at $80 \%$ of the total length of the TF domain from its left boundary (see Fig. 5)

Maxwell's equations 1 are solved with an explicit finitedifference time-domain (FDTD) scheme implicitly coupled with a Crank-Nicolson formulation for the electron momentum transfer equation 3. This numerical method is similar to the method described in Ref. ${ }^{4}$. Convolutional perfectly matching layer (CPML) absorbing boundary conditions are implemented in order to minimize reflections into the domain. Concerning plasma equations both fluid models, 4 and 10, are written in a nonconservative form and solved with a central difference scheme explicitly integrated in time with an Euler scheme. The microwave-plasma coupling is described as follows: the microwave solution obtained during one wave cycle yields the time-average electron power absorption (or the rms electric field) which is injected in the non- 


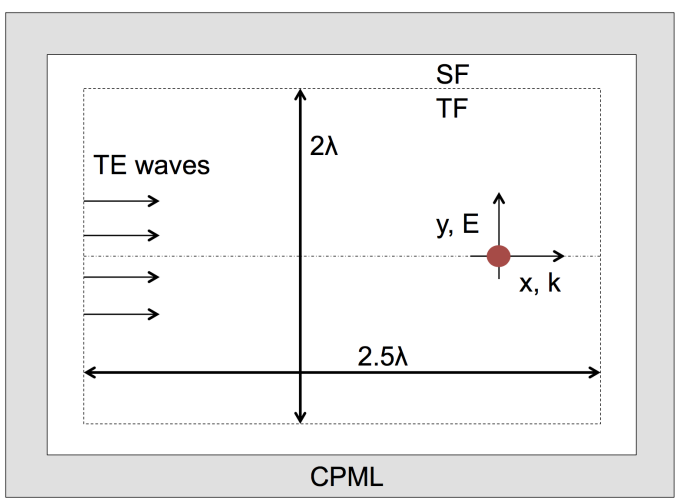

FIG. 5. 2D simulation domain and dimensions.

local plasma model (or local plasma model) to simulate the evolution of the plasma density over the next wave cycle. The plasma density is then injected back into the microwave equations to update the power absorption (or the rms electric field). In this procedure we make use of two different time steps, one describing the electromagnetic waves propagation $\left(\Delta t_{M}\right)$ and the other one the plasma evolution $\left(\Delta t_{p}\right)$. They are linked in the following way: $\Delta t_{M}=\Delta t_{p} / n$, where $n$ is a non-zero even integer and $\Delta t_{p}$ is set equal to the wave period according to our coupling strategy (i.e. $\left.\Delta t_{p}=T_{M}\right)$ ). Although the good practicality of the explicit formulation such a time stepping imposes CFL-like time step restrictions either for microwave and plasma problems. Concerning the latter, the restriction on the time step is more problematic with respect to the microwave case. Indeed during simulations it can often happen that in some points of the computational domain the maximum allowed plasma time steps become smaller than the wave period making the plasma explicit scheme unstable. Particularly the two-dimensional time step restrictions for the local and non-local models are (according to the Von-Neumann criterion):

$$
\begin{aligned}
& \Delta t_{p, \text { local }} \leq \frac{1}{2 D_{\text {eff }}}\left(\frac{1}{\frac{1}{\Delta x^{2}}+\frac{1}{\Delta y^{2}}}\right) \\
& \Delta t_{p, \text { non-local }} \leq \frac{1}{\frac{10}{3} D_{\text {eff }}}\left(\frac{1}{\frac{1}{\Delta x^{2}}+\frac{1}{\Delta y^{2}}}\right)
\end{aligned}
$$

where $\Delta x$ and $\Delta y$ are the spatial step sizes. We find that the allowed time steps depend on the square of the spatial step sizes (which in our case there is a unique spatial step size, $\Delta x=\Delta y=h$, as the grid is Cartesian) and the electron temperature, the microwave and gas conditions via the diffusion coefficient. In the nonlocal model the time condition is more restrictive than that of the local model because of the electron energy equation. Therefore to overcome plasma stability issues while keeping an explicit formulation a sub-cycling technique is adopted. This technique allows to express an explicit time integration with a given time step $\Delta t_{p}$ as suc- cessive explicit time integrations with smaller time steps $\Delta t_{p, i}\left(\right.$ where $\left.\Delta t_{p}=\sum_{i} \Delta t_{p, i}\right)$.

Further attention must be paid on the explicit formulation of the non-local model. Effectively the source term of the electron energy density equation 10 depends on the mean electron energy, more specifically $\Theta$ is a nonlinear rising function of $\varepsilon_{e}$. An explicit evaluation of that source term tends to cause instabilities in the solution (precisely on the mean electron energy) and consequently in the whole system of equations. Therefore as recommended by Hagelaar et al..$^{13}$ we adopt an implicit technique to treat the energy source term. The idea is to linearize the entire source term $(S)$ with respect to the mean electron energy (truncating the linearization at first order) as follows: $S^{t+1}=S^{t}+\partial_{\varepsilon_{e}} S^{t}\left(\varepsilon_{e}^{t+1}-\varepsilon_{e}^{t}\right)$, where superscripts represent the time at which variables are calculated. In our case, as the electron energy equation is solved for the electron energy density $n_{\varepsilon}$ the linearization is consequently adapted. The precise implicit expression of our energy source term $S=-n_{e}\left(e<\tilde{\mathbf{u}}_{e} \cdot \tilde{\mathbf{E}}>_{T_{M}}+\Theta\right)$ is:

$$
\begin{aligned}
S^{t+1}= & -n_{e}^{t}\left[e\left(<\tilde{\mathbf{u}}_{e} \cdot \tilde{\mathbf{E}}>_{T_{M}}\right)^{t}+\Theta^{t}\right]+ \\
& -\left(\frac{\partial \Theta}{\partial \varepsilon_{e}}\right)^{t}\left[\left(n_{e} \varepsilon_{e}\right)^{t+1}-n_{e}^{t+1} \varepsilon_{e}^{t}\right]
\end{aligned}
$$

where only the second term of $S, \Theta$, is linearized for the reason explained above. Also the electron density source term can be treated in the same way when the recombination coefficient is present.

In this paper we are studying the dynamics of $2 \mathrm{D}$ plasma filaments in air at different microwave and gas conditions. The characteristic length of the propagating plasma front $L$ can be extremely small (on the order of few micrometers). Moreover as the plasma filaments are in the simulation domain the electric field can present very large gradients at the tip of filaments. Therefore, we opt for using a fixed grid fine enough to resolve both plasma density and field gradients. Both plasma and Maxwell's equations are solved on the same grid of constant spacing $h=\lambda / n_{p}$. Since we are comparing models of different orders at different simulation conditions the parameter $n_{p}$ is determined as function of the "theoretical" $L$ at the beginning of each different simulation (for theoretical it is meant the value of $L$ corresponding to the incident $E_{r m s} / N$ at given $\left.\omega / N\right)$ in order to assure an appropriate, constant spatial resolution $L / h=$ const $\left(n_{p}\right.$ is a non-zero positive integer such that $\left.h \ll L\right)$ between simulations. The numerical solver has been efficiently parallelized with Message Passing Interface (MPI) library.

\section{NUMERICAL RESULTS}

In this section we assess the physical predictions of the local effective field and local mean energy models and 
the uncertainty associated with the choice of transport and rate coefficients. We present results for four sets of conditions. The wave frequency is kept the same and equal to $F=110 \mathrm{GHz}$ in the four cases.

- Case 1: $E=5 \mathrm{MV} / \mathrm{m}, p=710$ torr

- Case 2: $E=5 \mathrm{MV} / \mathrm{m}, p=400$ torr

- Case 3: $E=5 \mathrm{MV} / \mathrm{m}, p=200$ torr

- Case 4: $E=2.82 \mathrm{MV} / \mathrm{m}, p=400$ torr

The rms values are respectively $3.54 \mathrm{MV} / \mathrm{m}$ and 1.992 $\mathrm{MV} / \mathrm{m}$ for the field amplitudes of $5 \mathrm{MV} / \mathrm{m}$ and 2.82 $\mathrm{MV} / \mathrm{m}$. Several microwave and gas conditions may be chosen for performing the simulations, but only some characteristic conditions would be necessary to make a representative comparison. For this purpose two particular scenarios are identified. The $E_{r m s} / N$ values are 154.7 Td, 274.6 Td, 549.2 Td, 154.7 Td respectively for cases 1 to 4 .

In the first three cases, the wave field $E$ is fixed and the gas density gradually decreases. This scenario actually corresponds to the situation when the gas dynamics is taken into account and the gas heating effects act on the plasma dynamics. In the last case the strength of the incident electric field and gas density are simultaneously decreased such that $E_{r m s} / N$ is the same as in case 1 $\left(E_{r m s} / N=154.7 \mathrm{Td}\right)$.

The conditions above are similar to those of experiments of Ref. ${ }^{9}$ and Ref. ${ }^{24}$. As experimentally observed, in this gas pressure range the microwave discharge still has a streamer nature manifesting some early diffuse characteristics at lower pressures. From previous studies we are fully aware that the formation and propagation of a selforganized pattern of microwave filaments in overcritical field conditions are essentially due to the combination of wave scattering and ionization-diffusion mechanisms.

We expect that the local effective field approximation becomes inaccurate when the macroscopic length scales of the plasma become small with respect to the energy relaxation length. This should start to happen from case 1 to case 3 since the reduced electric field increases, leading to larger plasma density gradients and, possibly, large variations of the electric field over the energy relaxation length. Therefore the differences between results from the local effective field and local mean energy models should increase when the gas density is decreased at constant applied field and frequency. On the other hand, keeping the ratio $E_{r m s} / N$ constant while decreasing the gas density leads to a simple scaling of the plasma dynamics as the width of the plasma front increases as the energy relaxation length (both scale as $1 / N$ ). Therefore the differences between local mean energy approximation and local effective field approximation should not strongly change between case 1 and case 4 (the variation of $\omega / N$ between case 1 and case 4 should not have a strong incidence on the results).
We can identify on Fig. 4 the variations of the nonlocality parameter $\delta$ in the four cases. From case 1 to case $3, E_{r m s} / N$ and $\omega / N$, both increase as $N$ decreases so we diagonally move over a straight line on the $2 \mathrm{D}$ plot. As a result, $\delta$ increases from $\delta=0.54 \times 10^{-2}$ for case 1 , to $\delta=10.37 \times 10^{-2}$ for case 3 . Comparing case 1 and case 4 , we move over a horizontal straight line corresponding to a constant value of $E_{r m s} / N$. The parameter $\delta$ decreases slightly (because of the change in $\omega / N$ ), from $\delta=0.54 \times$ $10^{-2}$ to $\delta=0.48 \times 10^{-2}$.

Figure 6 shows the space distribution of the plasma density of the considered microwave discharge at 5 $\mathrm{MV} / \mathrm{m}, 710$ torr (case 1) after $100 \mathrm{~ns}$, obtained from the local and non-local models (as reference, in this case the grid spatial resolution is set equal to $\lambda / 800$, see $\mathrm{V}$ ). The well-known "fish-bone" structure of the discharge is observable. In this case, the parameter $\delta$ is about $5 \times 10^{-3}$ (see Fig. 4) and the simulation results are very similar for the local effective field and local mean energy models. The propagation of plasma front is described in a similar manner by the two models (Fig. 7). In such conditions the electron energy transport over the plasma non-uniformities is small and the local mean energy approximation tends to the local effective field approximation.

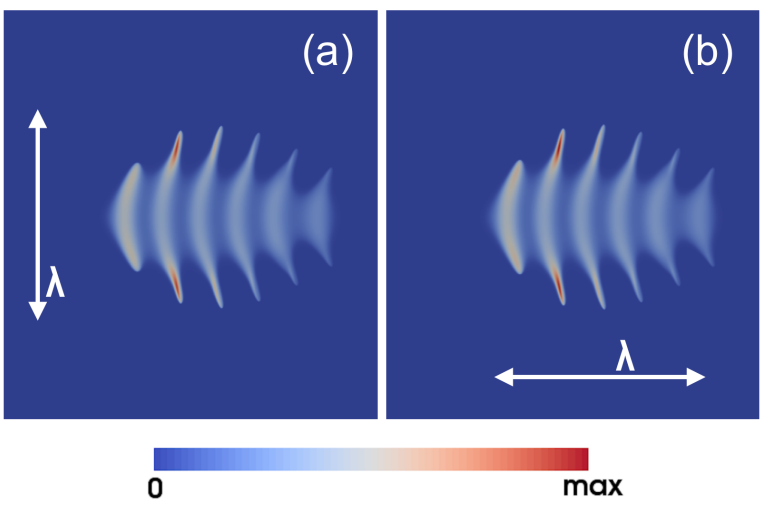

FIG. 6. (Color online) Space distribution of the plasma density at $100 \mathrm{~ns}$ for a $110 \mathrm{GHz}, 5 \mathrm{MV} / \mathrm{m}$ amplitude of the incident wave (from left to right) at 710 torr. (a) is the result of the local model and (b) the result of the non-local model. The maximum value of the plasma density are $4.72 \times 10^{21}$ $\mathrm{m}^{-3}$ (a) and $5.14 \times 10^{21} \mathrm{~m}^{-3}(\mathrm{~b})$.

We now look at the effects, in the same conditions, of some approximations on the data used in the simulations. In the papers by Chaudhury et al. ${ }^{4-6}$, the electron temperature in the diffusion coefficient was taken as a constant, equal to $2 \mathrm{eV}$ (see III). Figure 8 shows a comparison of the results obtained with the local model with a constant electron temperature of $2 \mathrm{eV}$ in the diffusion coefficient and with a temperature dependence obtained from BOLSIG+ (3). The spatial profiles are similar but the velocity of the plasma front is about $20 \%$ lower in the constant temperature case. This is not surprising since the theoretical velocity of the plasma front is pro- 

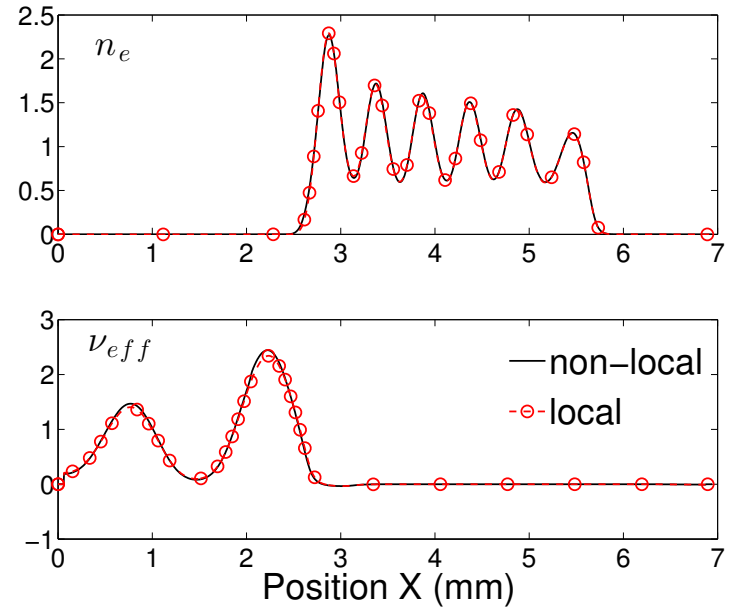

FIG. 7. (Color online) Distributions along the central X-axis of the plasma density and the effective ionization frequency in conditions of Fig. 6 obtained from the local (red dashed line) and non-local (black solid line) models. The units are $10^{21}$ $\mathrm{m}^{-3}$ for the density and $10^{9} \mathrm{~s}^{-1}$ for the effective frequency.

portional to the square root of the electron diffusion coefficient in the plasma front, which is a function of electron temperature.
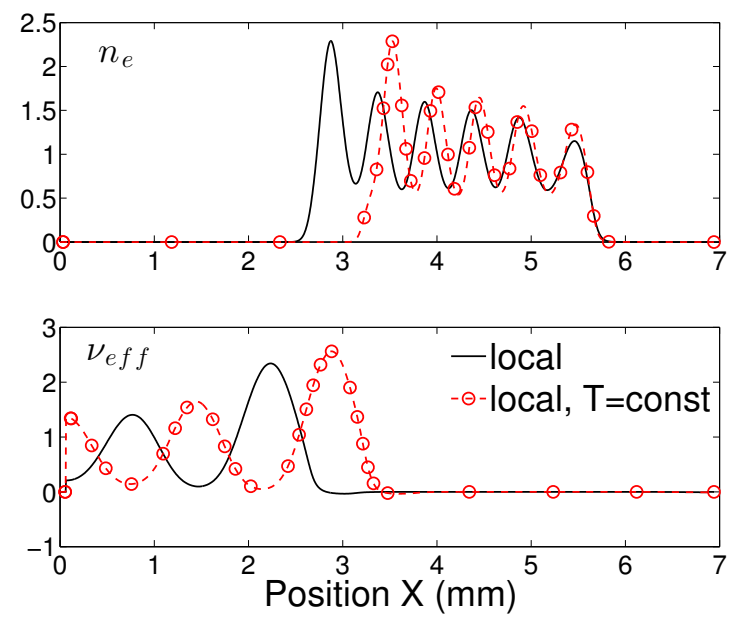

FIG. 8. (Color online) Distributions along the central X-axis of the plasma density and the effective ionization frequency in conditions of Fig. 6 obtained from the local model assuming the electron temperature variable (black solid line) and constant $T_{e}=2 \mathrm{eV}$ (red dashed line). The units are $10^{21} \mathrm{~m}^{-3}$ for the density and $10^{9} \mathrm{~s}^{-1}$ for the effective frequency.

Actually, the electron temperature distribution given by the non-local model is far from uniform as can be seen on Fig. 9. We can also expect that the constant temperature model will strongly underestimate the velocity of the streamer elongation.

By decreasing the gas density and keeping constant all other parameters (going from case 1 to case 3 ), the dis-

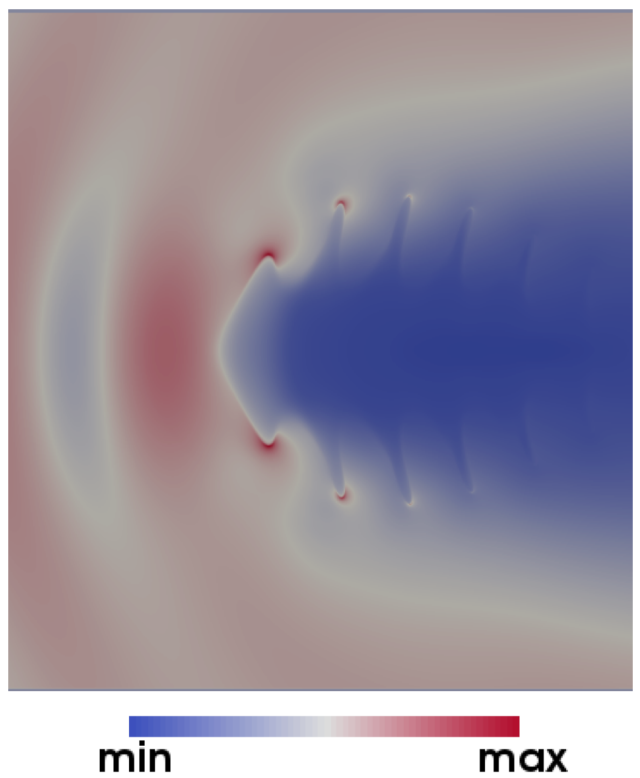

FIG. 9. (Color online) Space distribution of the electron temperature obtained from the non-local model in conditions of Fig. 6. The color scale is between the minimum value of 0.58 $\mathrm{eV}$ and the maximum value of $4.77 \mathrm{eV}$.

crepancy between the two models gradually should grow according to our parameter $\delta$. Figures 10 (a) and 10(b) display the space distribution of the plasma density at 400 torr and same incident field amplitude of Fig. 6 obtained from the local and non-local models. In this condition $\left(\delta\right.$ is about $\left.3 \times 10^{-2}\right)$ the discharge propagation is faster than in the previous case since almost the whole simulation domain is occupied by the plasma in 30 ns (the previous case, in Fig. 6, was shown at time $t=100 \mathrm{~ns})$. Even though the spatial profiles of plasma obtained by the two models are qualitatively the same, we see that the plasma in the non-local model propagates a little faster both in the $X$ and $Y$ directions. If the electron temperature is fixed at $2 \mathrm{eV}$ during the simulation as in previous papers, the local model does not predict at all the same dynamics (different plasma space distribution and slower front propagation of more than $35 \%$, Fig. 10(c)) as the local model with suitable temperature. In fact the electron temperature reaches values of about $6.53 \mathrm{eV}$ (according to the estimation given by the non-local model) because of higher reduced electric field compared to the atmospheric case. Note that the simulation performed with a constant electron momentum transfer frequency shown in Fig. 10(d) exhibits only small differences with the case of Fig. 10(a).

At 200 torr and the same wave field and frequency as above the discrepancy between the two approximations becomes more evident. Since the energy transport plays a more important role in such a condition ( $\delta$ is about 0.1 ) the local model presents an even slower plasma propagation compared to the non-local model. Figure 11 shows the space distribution of the plasma density at 200 torr 

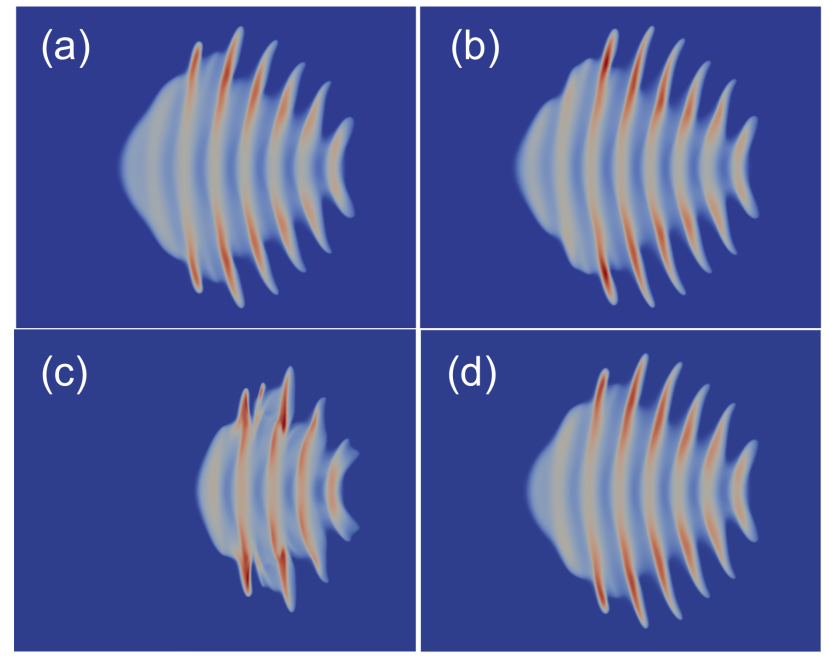

FIG. 10. (Color online) Space distribution of the plasma density at $30 \mathrm{~ns}$ for a $110 \mathrm{GHz}, 5 \mathrm{MV} / \mathrm{m}$ amplitude of the incident wave (from left to right) at 400 torr obtained from the local (a) and non-local (b) models, local model assuming the electron temperature constant, $T_{e}=2 \mathrm{eV}$ (c) and local model assuming the momentum transfer collision frequency constant, $\nu_{m}=5.3 \times 10^{9} p(\mathrm{~d})$, where $p=400$ torr. For (a), (b), (c) and (d) same color bar as Fig. 6 with maximum values of $4.22 \times 10^{21} \mathrm{~m}^{-3}, 4.76 \times 10^{21} \mathrm{~m}^{-3}, 5.20 \times 10^{21} \mathrm{~m}^{-3}$ and $4.25 \times 10^{21} \mathrm{~m}^{-3}$, respectively. The computational domain represented in figure is exactly $2.5 \lambda \times 2 \lambda$.

after 9 ns obtained from the two fluid approximations. Note that the ionization rate is much faster than in the previous cases because of the higher reduced electric field.
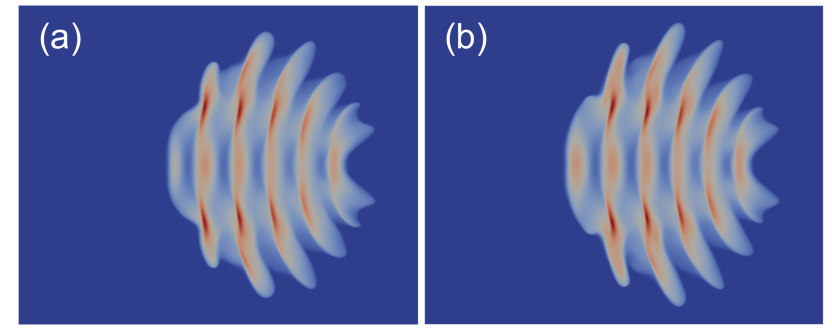

FIG. 11. (Color online) Space distribution of the plasma density at $9 \mathrm{~ns}$ for a $110 \mathrm{GHz}, 5 \mathrm{MV} / \mathrm{m}$ amplitude of the incident wave (from left to right) at 200 torr. (a) is the result of the local model and (b) the result of the non-local model. Same color bar as Fig. 6 with maximum values of $4.75 \times 10^{21} \mathrm{~m}^{-3}$ (a) and $4.80 \times 10^{21} \mathrm{~m}^{-3}$ (b). The computational domain represented in figure is exactly $2.5 \lambda \times 2 \lambda$.

On the other hand, if the incident electric field strength is reduced with decreasing the gas pressure such that the ratio $E_{r m s} / N$ is kept constant (case 1 and case 4 ), $\delta$ slightly decreases (see Fig. 4), i.e. the discrepancy between the models remains minimal. A precise simulation at 400 torr confirms the above statement and the results are shown in Fig. 12 and Fig. 13. In this second scenario, conditions are such that the estimation of the electron energy (hence the electron temperature) from a non-local enery equation is not necessarily required for a correct description of the ionization process. Moreover, as experimentally observed at 400 torr the filamentary structure is smeared out.
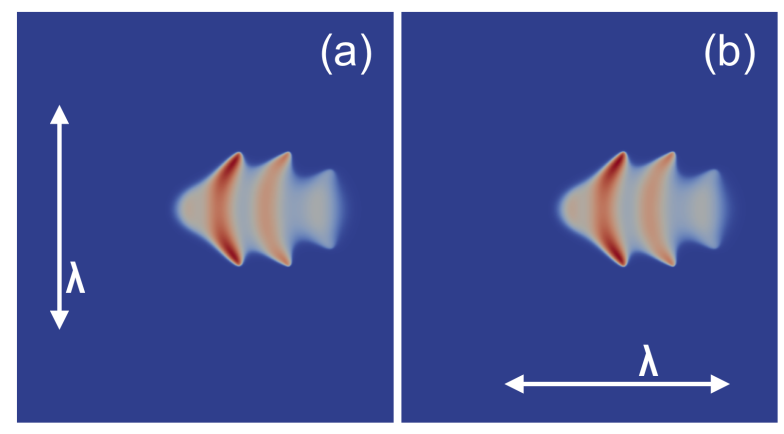

FIG. 12. (Color online) Space distribution of the plasma density at $100 \mathrm{~ns}$ for a $110 \mathrm{GHz}, 2.82 \mathrm{MV} / \mathrm{m}$ amplitude of the incident wave (from left to right) at 400 torr. (a) is the result of the local model and (b) the result of the non-local model. Same color bar as Fig. 6 with maximum values of $1.27 \times 10^{21}$ $\mathrm{m}^{-3}$ (a) and $1.28 \times 10^{21} \mathrm{~m}^{-3}$ (b).
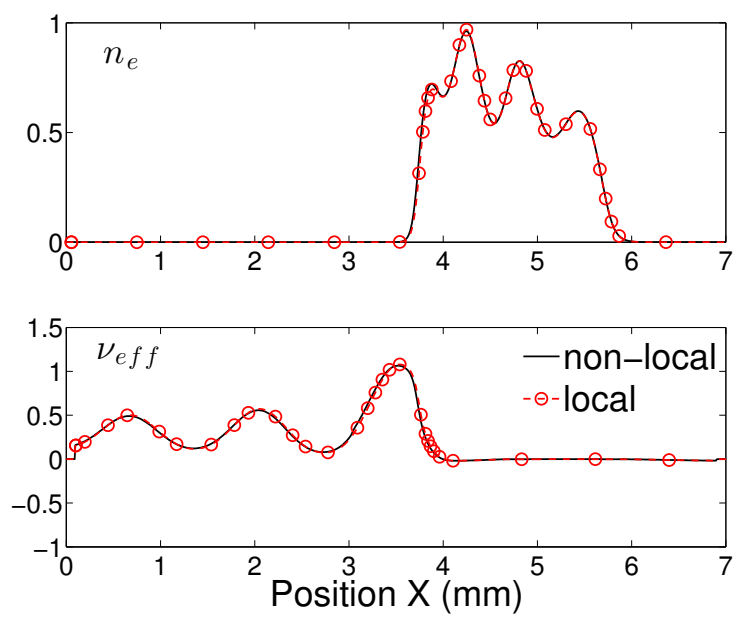

FIG. 13. (Color online) Distributions along the central X-axis of the plasma density and the effective ionization frequency in conditions of Fig. 12 obtained from the local (red dashed line) and non-local (black solid line) models. The units are $10^{21}$ $\mathrm{m}^{-3}$ for the density and $10^{9} \mathrm{~s}^{-1}$ for the effective frequency.

In order to quantify discrepancy between the local and non-local models and verify the theoretical concept of the dimensionless parameter $\delta$ we focus on the comparison of the plasma front velocities along the central $\mathrm{X}$-axis of the computational domain. The plasma front velocity is obtained by recording in time the position of a point in the front where the plasma density reaches a constant value. This value is calculated as a percentage (always the same between all simulations) of the maximum central X-axis plasma density reached in the related simulation. The 
position of the plasma front as function of time obtained from the models for all above conditions are shown in Fig. 14. Furthermore a relative error between the average velocity $V_{f}$ obtained from the non-local model and from the local model, $V_{f, \text { local }}$ is estimated, $\epsilon=\left|V_{f}-V_{f, \text { local }}\right| / V_{f}$, and reported in Table I for the different cases considered here.
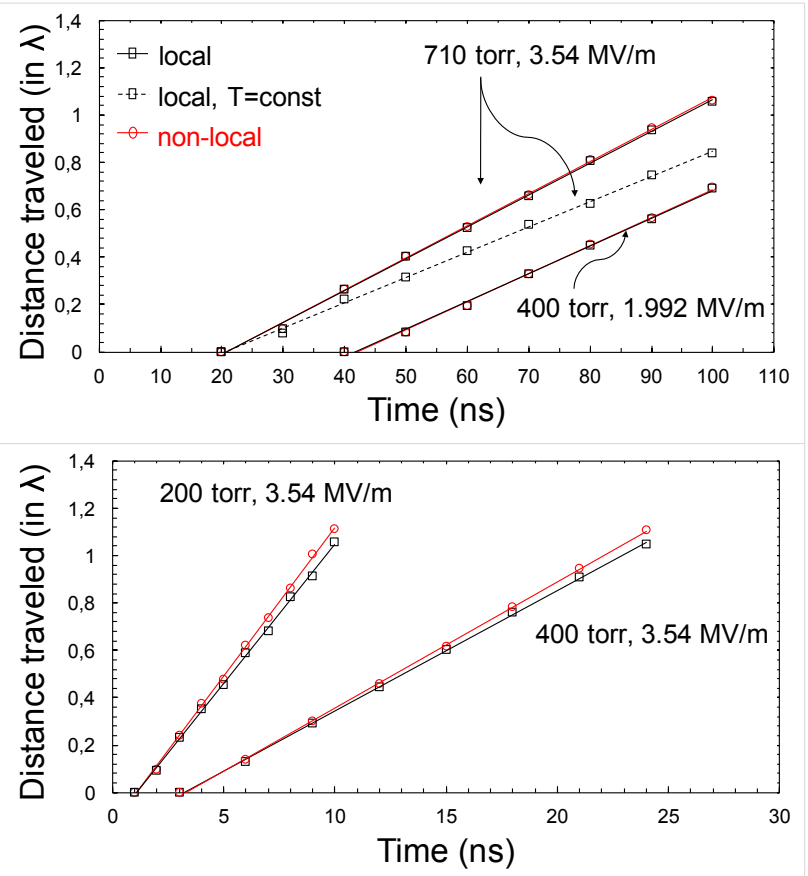

FIG. 14. (Color online) Position of the plasma front along the central $\mathrm{X}$-axis as function of time obtained from the nonlocal model (solid line with circle markers in red), local model (solid line with square markers in black) and local model with a constant electron temperature, $T_{e}=2 \mathrm{eV}$ (dashed line with square markers in black) at the above simulation conditions (the rms value of the electric field is reported in the figures). Points are plotted corresponding to position where the plasma density reaches values of $10^{19} \mathrm{~m}^{-3}$ at 710 torr, $1.32 \times 10^{19} \mathrm{~m}^{-3}$ at 400 torr, $1.43 \times 10^{19} \mathrm{~m}^{-3}$ at 200 torr and $7.37 \times 10^{18} \mathrm{~m}^{-3}$ at 400 torr and $E_{r m s}=1.992 \mathrm{MV} / \mathrm{m}$.

The plots of Fig. 14 and the results reported in Table I illustrate the discrepancies between the local effective field and local mean energy models (or local and non-local models) as $E / N$ increases (cases 1 to 3 ), or for the same $E / N$ at two different pressures. We see the differences between the two models are very small for case 1 and increases to less than $7 \%$ for case 3 . For case 4 , the differences in front velocity between the two models is less than $1 \%$. The front velocity for case 1 and case 4 are very similar. This is because $E / N$ is the same for both cases. The pressure in case 4 is lower than for case 1 but position and time scale practically as $1 / N$ and the front velocities are similar. The slight difference being the two cases is due to recombination (a non-linear process that does not follow similarity laws) and to the different values of $\omega / N$.
Finally we note on Fig. 14 that the results for case 1 with a constant electron temperature give a significally smaller propagation velocity $29.2 \mathrm{~km} / \mathrm{s}$, instead of 37.1 $\mathrm{km} / \mathrm{s}$. The front velocity for case 1 and for the ionization frequency used in the paper of Chaudhury et al..$^{5}$ (see Fig. 1) was also much smaller (on the order of $10 \mathrm{~km} / \mathrm{s}$ ). This shows that the results are quite sensitive to the transport coefficients and rate coefficients used in the model and that the errors due to these data may be larger than the errors due to the local effective field approximation in our conditions. Compared with experimental results ${ }^{7}$ the model result of $37.1 \mathrm{~km} / \mathrm{s}$ for case 1 is of the same order of magnitude as the measured one, but a detailed comparison is difficult as discrepancies may result due to uncertainty on the exact electric field intensity and the different spatial profile of the electric field used in the experimental configurations, the strong dependence of the ionization coefficient with the electric field and the 3D nature of the discharge. However the theoretical front velocity $V_{t h}=2\left(\nu_{i} D_{e}\right)^{1 / 2}$, calculated for a constant field equal to the incident field, as a function of the gas pressure shows the same trend as the simulation velocity, e.g. for cases 1, 2 and 3 the values of $V_{t h}$ are $20.6 \mathrm{~km} / \mathrm{s}, 73.3$ $\mathrm{km} / \mathrm{s}$ and $188,4 \mathrm{~km} / \mathrm{s}$ respectively (for comparison see Table I, note that this velocity is lower than the simulation velocity because of a larger time averaged field in the front with respect to the incident field ${ }^{5}$ ).

TABLE I. Average plasma front velocity $V_{f}$ from the local mean energy model and relative errors $\epsilon$ (in percentage) of the front velocity calculated with the local effective field model (shown in Fig. 14), for the four different cases. The value of the non-locality parameter $\delta$ is also indicated.

\begin{tabular}{lccc|c|c}
\hline & $\begin{array}{c}\delta \\
\left(\times 10^{-2}\right)\end{array}$ & $\begin{array}{c}E_{r m s} \\
(\mathrm{MV} / \mathrm{m})\end{array}$ & $\begin{array}{c}\text { Pressure } \\
\text { (torr) }\end{array}$ & $\begin{array}{c}V_{f} \\
(\mathrm{~km} / \mathrm{s})\end{array}$ & $\begin{array}{c}\epsilon \\
(\%)\end{array}$ \\
\hline Case 1 & 0.54 & 3.54 & 710 & 37.1 & 1.47 \\
Case 2 & 3.07 & 3.54 & 400 & 144.2 & 4.54 \\
Case 3 & 10.37 & 3.54 & 200 & 342. & 6.45 \\
Case 4 & 0.48 & 1.992 & 400 & 32.2 & 0.85 \\
\hline
\end{tabular}

\section{CONCLUSION}

We have presented in this paper a study of the accuracy of models of the plasma dynamics during microwave breakdown at high pressure (above 200 torr) and high frequency $(110 \mathrm{GHz})$. We have compared the predictions of models based on the local effective field ("local model") and local mean energy ("non-local model") approximations. We have first defined a parameter $\delta$ that characterizes the "non-locality" of the problem. The numerical results confirm the increase of the discrepancy between local and non-local model as $\delta$ increases. For increasingly over-critical (i.e. above breakdown) microwave fields the error due to the local effective field approximation increases. However we find that this error is not as large as could be expected. For example the relative er- 
ror in the velocity of the plasma front predicted by the local model is less than $7 \%$ for an electric field of amplitude as large as $5 \mathrm{MV} / \mathrm{m}$ at 200 torr. Such large values of $E / p$ (or $E / N)$ are possible during the process of gas heating by the microwave field, since the gas density can significantly decrease ${ }^{19,24}$. The local mean energy approximation is therefore recommended for such studies. The fact that the local effective field approximation, in spite of its simplicity, appears to be a reasonably good approximation in a relatively large range of conditions is consistent with the analysis of dc streamer propagation by Markosyan et al. ${ }^{22}$. This work also shows that in the conditions considered in this paper the errors in the results are more likely due to inaccuracies in the transport and rate coefficients used in the simulations than to the local effective field approximation.

\section{ACKNOWLEDGEMENTS}

This research is supported by the French ministry of defense (Direction Géneŕale de l'Armement, DGA). E.A. benefits from an ONERA/DGA fellowship. The authors are grateful to Dr. G.J.M. Hagelaar for the helpful discussions.

\section{REFERENCES}

${ }^{1}$ J. A. Bittencourt. Fundamentals of plasma physics. Springer, New York, third edition, 2004.

${ }^{2}$ J. P. Boeuf, B. Chaudhury, and G. Q. Zhu. Theory and modeling of self-organization and propagation of filamentary plasma arrays in microwave breakdown at atmospheric pressure. Phys. Rev. Lett., 104(1):015002, 2010.

${ }^{3}$ J. P. Boeuf and L. C. Pitchford. Two-dimensional model of a capacitively coupled rf discharge and comparisons with experiments in the gaseous electronics conference reference reactor. Phys. Rev. E, 51(2), 1995.

${ }^{4}$ B. Chaudhury and J. P. Boeuf. Computational studies of filamentary pattern formation in a high power microwave breakdown generated air plasma. Plasma Science, IEEE Transactions on, 38(9):2281-2288, 2010.

${ }^{5}$ B. Chaudhury, J. P. Boeuf, and G. Q. Zhu. Pattern formation and propagation during microwave breakdown. Phys. Plasmas, 17(9):123505, 2010.

${ }^{6}$ B. Chaudhury, J. P. Boeuf, G. Q. Zhu, and O. Pascal. Physics and modeling of microwave streamers at atmospheric pressure. J. Appl. Phys., 110(9):113306, 2011.

${ }^{7}$ A. M. Cook, J. S. Hummelt, M. A. Shapiro, and R. J. Temkin. Observation of plasma array dynamics in $110 \mathrm{ghz}$ millimeter-wave air breakdown. Phys. Plasmas, 18(10):100704, 2011.

${ }^{8}$ A. M. Cook, J. S. Hummelt, M. A. Shapiro, and R. J. Temkin. Millimeter wave scattering and diffraction in 110 ghz air breakdown plasma. Phys. Plasmas, 20(4):043507, 2013.

${ }^{9}$ A. M. Cook, M. A. Shapiro, and R. J. Temkin. Pressure dependence of plasma structure in microwave gas breakdown at 110 ghz. Appl. Phys. Lett., 97(1):011504, 2010.

${ }^{10}$ G. K. Grubert, M. M. Becker, and D. Loffhagen. Why the local-mean-energy approximation should be used in hydrody- namic plasma descriptions instead of the local-field approximation. Phys. Rev. E, 80(3), 2009.

${ }^{11}$ G. J. M. Hagelaar, K. Makasheva, L. Garrigues, and J. P. Boeuf. Modelling of a dipolar microwave plasma sustained by electron cyclotron resonance. J. Phys. D: Appl. Phys., 42(19), 2009.

${ }^{12}$ G. J. M. Hagelaar and L. C. Pitchford. Solving the boltzmann equation to obtain electron transport coefficients and rate coefficients for fluid models. Plasma Source Science Technology, 14(4), 2005.

${ }^{13}$ G. J. M. Hagelaar and Kroesen M. W. Speeding up fluid models for gas discharges by implicit treatment of the electron energy source term. J. Comput. Phys., 59:1-12, 2000.

${ }^{14}$ Y. Hidaka, E. M. Choi, I. Mastovsky, M. A. Shapiro, J. R. Sirigiri, and R. J. Temkin. Observation of large arrays of plasma filaments in air breakdown by $1.5-\mathrm{mw} 110$-ghz gyrotron pulses. Phys. Rev. Lett., 100(3), 2008.

${ }^{15}$ Y. Hidaka, E. M. Choi, I. Mastovsky, M. A. Shapiro, J. R. Sirigiri, R. J. Temkin, G. F. Edmiston, A. A. Neuber, and Y. Oda. Plasma structures observed in gas breakdown using a $1.5 \mathrm{mw}$, 110 ghz pulsed gyrotrona). Phys. Plasmas, 16(5):055702, 2009.

${ }^{16}$ J. S. Hummelt, M. A. Shapiro, and R. J. Temkin. Spectroscopic temperature measurements of air breakdown plasma using a 110 ghz megawatt gyrotron beam. Phys. Plasmas, 19(12), 2012.

${ }^{17}$ I. A. Kossyi, A. Y. Kostinsky, A. A. Matveyev, and V. P. Silakov. Kinetic scheme of the non-equilibrium discharge in nitrogen-oxygen mixtures. Plasma Sources Science and Technology, 1:207-220, 1992.

${ }^{18}$ K. Kourtzanidis, J. P. Boeuf, and F. Rogier. Three dimensional simulations of pattern formation during high-pressure, freely localized microwave breakdown in air. Phys. Plasmas, 21(12):123513, 2014.

${ }^{19} \mathrm{~K}$. Kourtzanidis and L. L. Raja. On the accuracy of the rate coefficients used in plasma fluid models for breakdown in air. Phys. Plasmas, 23:074503, 2016.

${ }^{20} \mathrm{~K}$. Kourtzanidis, F. Rogier, and J. P. Boeuf. Gas heating effects on the formation and propagation of a microwave streamer in air. J. Appl. Phys., 118(10):103301, 2015.

${ }^{21}$ A. D. MacDonald. Microwave breakdown in gases. Wiley, New York, first edition, 1966

${ }^{22}$ A. H. Markosyan, J. Teunissen, S. Dujko, and U. Ebert. Comparing plasma fluid models of different order for 1d streamer ionization fronts. Plasma Sources Science and Technology, 24(4), 2015.

${ }^{23}$ S. K. Nam and J.P. Verboncoeur. Theory of filamentary plasma array formation in microwave breakdown at near-atmospheric pressure. Phys. Rev. Lett., 103(5), 2009.

${ }^{24}$ S. C. Schaub, J. S. Hummelt, W. C. Guss, M. A. Shapiro, and R. J. Temkin. Electron density and gas density measurements in a millimeter-wave discharge. Phys. Plasmas, 23:083512, 2016.

${ }^{25}$ BOLSIG+ solver. https://www.bolsig.laplace.univ-tlse.fr/.

${ }^{26}$ M. Takahashi, Y. Kageyama, and N. Ohnishi. Joule-heatingsupported plasma filamentation and branching during subcritical microwave irradiation. AIP Advances, 7(5):055206, 2017.

${ }^{27}$ Biagi v8.9 database. www.lxcat.net, retrieved on September 1, 2016.

${ }^{28} \mathrm{~W}$ Woo and J. S. DeGroot. Microwave absorption and plasma heating due to microwave breakdown in the atmosphere. Phys. Fluid, 27:475, 1984.

${ }^{29}$ W. Yang, Q. Zhou, and Z. Dong. Simulation study on nitrogen vibrational and translational temperature in air breakdown plasma generated by $110 \mathrm{ghz}$ focused microwave pulse. Phys. Plasmas, 24(1), 2017.

${ }^{30}$ Q. Zhou and Z. Dong. Modeling study on pressure dependence of plasma structure and formation in $110 \mathrm{ghz}$ microwave air breakdown. Appl. Phys. Lett., 98(16), 2011.

${ }^{31}$ G. Q. Zhu, J. P. Boeuf, and B. Chaudhury. Ionization-diffusion plasma front propagation in a microwave field. Plasma Sources Science and Technology, 20(3):035007, 2011. 